\title{
Studies of properties of the seat suspension system with air spring
}

\author{
Piotr Woś ${ }^{1, *}$, Ryszard Dindorf ${ }^{2}$ \\ ${ }^{1}$ EDP Faculty of Mechatronics and Machine Design, Kielce University of Technology, Kielce, 25-314, Poland \\ ${ }^{2}$ EDP Faculty of Mechatronics and Machine Design, Kielce University of Technology, Kielce, 25-314, Poland
}

\begin{abstract}
This article presents issues related to the study of the seat vibration isolating properties of the working machine. The spring force of the suspension system is realized by means of a pneumatic spring. The mathematical model of the air spring is presented. The results of research presented in the form of power spectral density of the vibration acceleration of the suspension system.
\end{abstract}

\section{Introduction}

Machine operator, drivers are exposed to long-term vibration impact which can lead to the emergence of occupational disease - a vibration syndrome. Vibrations from machines affect people locally and are transmitted through the upper limbs, as well as through the substrate and act on the entire body. People performing work in a sitting position, ie: drivers, machines and construction vehicles operators and tractor drivers are exposed to general vibrations, which are transferred to the body from the ground through the pelvis and back. The range of vibration frequencies depends on individual human structure and ranges from $2-12 \mathrm{~Hz}$ for internal organs and chest. Tests on preventing these effects are carried out for many years by the use of newer technologies of suspensions of both entire machines and the working seats of vehicle operators [1], [4]. The basic methods of counteracting the negative effects of vibrations for machine and vehicle operators are the construction of seat suspension with vibration insulation properties [2]. These are solutions with passive and active vibration insulation. In the seats of machines with passive vibration insulation elements with linear elasticity and non-linear damping are used. In this type of suspension, the damping and elasticity coefficients are selected according to the prevailing working conditions [5]. In many constructions, there are used the solutions which allow to change the attenuation coefficient by the operator depending on the individual assessment of his weight and working conditions [6], [7]. Achieving of effective damping can be done automatically or manually by setting a constant pressure value in the pneumatic spring.

The task of modern suspension systems is, on one hand, to reduce resonance vibrations and ensure proper machine stability, and on the other hand, to isolate forced vibrations at higher frequencies [9]. Designing a primary or secondary suspension to meet these requirements for different types of surface on which the machine moves and with a limited suspension working space requires the adjustment of a number of parameters of this system. Typical, passive suspension is equipped with coil springs and shock absorbers with constant damping coefficient. The low value of the damping coefficient in such systems causes a significant strengthening of the resonant vibrations of the protected object with simultaneous favorable vibration isolation in the resonance range. In turn, the high value of the damping factor means the reduction of resonant vibrations, but also insufficient vibro-isolation at higher vibration frequencies.

\section{Air suspension system}

Bellows cylinders have found their application especially in applications where linear mass transfer is necessary and where it is necessary to eliminate vibrations, effectively solving the problem of vibration in many industrial applications [2], [8]. The pneumatic bellows have been designed for flexible assembly of vibration generating devices. The actuators are used in cars and trucks in the systems of pneumatic suspension of the car suspension, or for the purpose of elastic fixing of vehicles and trailers, which allows for isolation of vibrations and vibrations of vehicle components. Bellows cylinders used as pneumatic springs in connection with the weight of the load create vibrating systems.

\subsection{Air spring equation}

Bellows cylinders are used as executive elements in pneumatic drive systems of machines and devices and as pneumatic springs (cushions) in vibration isolation of machines, amortization of vehicles, etc. Bellows

* Corresponding author: wos@tu.kielce.pl 
actuators in pneumatic drives work as single-acting actuators - pushing actuators. The operation of these actuators consists of filling (applying pressure) and emptying (venting). From the point of view of vibroisolation, it is important to operate the bellows actuator after filling with constant pressure. Then it fulfills the function of an air spring.

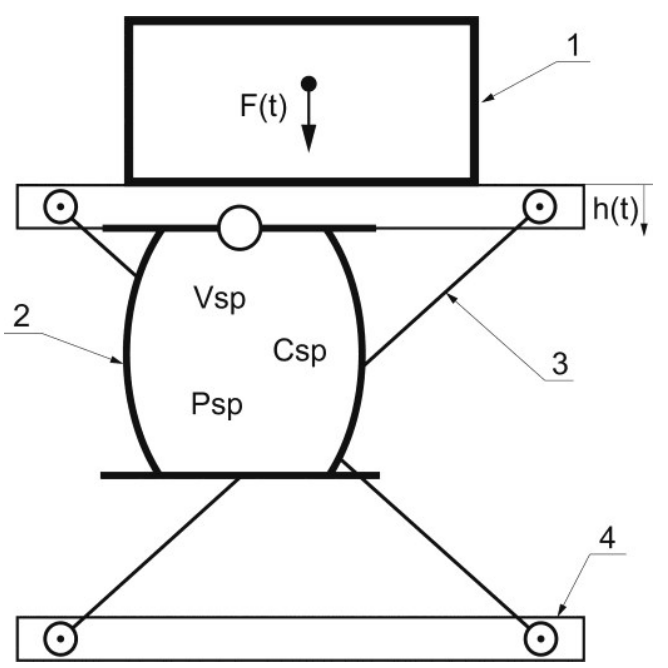

Fig.1. Scheme of air spring in suspension seat

The scheme of the vibration isolation system with the use of an air spring is shown in Figure 1. The system consists of mass (1) loading the system (3), causing mass vibrations in the vertical direction. The internal volume of the pneumatic spring (2) varies with the mass oscillation. This causes a change in the pressure in the spring.

The equation of motion according to the figure 1 can be written as follows:

$$
m \ddot{h}+c_{s p} h=F
$$

where: $h$ - deflection of air springs, $m$ - mass loading, $c_{s p}$ - stiffness bellows, $F$ - external force

The stiffness of the pneumatic bellow (air spring) is expressed by the ratio of the load $m$ to the deflection $h$ of the bellows $c_{s p}=d F / d h$, and the dependence on its deflection $h$ and pressure $p_{s p}$ are determined as follows:

$$
c_{s p}=\frac{d F}{d h}=\frac{d\left(p_{s p} A_{e}\right)}{d h}=A_{e} \frac{d p_{s p}}{d h}+p_{s p} \frac{d A_{e}}{d h}
$$

where: $p_{s p}$ - internal pressure, $A_{e}$ - effective surface of air spring

To determine the derivative $d p_{s p} / d h$ and assume adiabatic transformation were $\kappa=1.4$ is adiabatic coefficient. The change in pressure depending on the deflections of the bellows is:

$$
\frac{d\left(p_{s p} V_{s p}{ }^{\kappa}\right)}{d h}=\kappa p_{s p} V_{s p}{ }^{\kappa-1} \frac{d V_{s p}}{d h}+V_{s p}{ }^{\kappa} \frac{d p_{s p}}{d h}=0
$$

$$
\frac{d p_{s p}}{d h}=-\frac{p_{s p} \kappa}{V_{s p}} \frac{d V_{s p}}{d h}
$$

With the increase of pressure $p_{s p}$, the height of the spring increases and at the same time the volume $V_{s p}$ increases too. Taking into account the dependence $d V_{s p} / d h \approx-A_{e}$ and (4) we get the following equation:

$$
\frac{d p_{s p}}{d h}=-A_{e} \frac{p_{s p} \kappa}{V_{s p}}
$$

As a result, the stiffness of the air spring depending on its deflection $h$ and gas capacity $C g$ and pressure $p_{s p}$ was determined as follows:

$c_{s p}(h, p)=A_{e}^{2} \frac{p_{s p} \kappa}{V}+p_{s p} \frac{d A_{e}}{d h}=\frac{A_{e}^{2}}{C_{g}}+p_{s p} \frac{d A_{e}}{d h}=c_{s p 1}+c_{s p 2}$

where: $C_{g}$ - gas capacity, $C_{g}=V_{s p} / K_{g}, K_{g}$ - bulk modulus of elasticity of air, $K_{g}=\kappa p_{s p}, \kappa=1.4$ adiabatic exponent.

The second $c_{s p 2}$ component of the equation shows the change in the effective surface of the pneumatic spring We get a classic model of an air spring according to the drawing (fig.2).

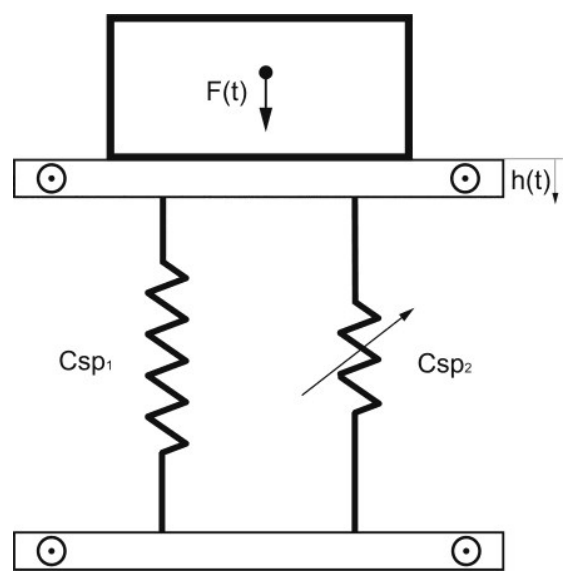

Fig.2. Air spring model

Assuming that the change in the airbag volume is:

$$
V_{s p}=V_{s p_{0}}-\Delta V_{s p}=V_{s p_{0}}-A_{e} \cdot h
$$

and the assumption that $V_{s p_{0}}=A_{e} h_{0}$ it was then that the stiffness of a flat was defined as:

$$
c_{s p}(h)=\frac{\kappa \cdot p_{s p 0} \cdot A_{e}}{h_{0}}\left(\frac{V_{s p_{0}}}{h_{0}-h}\right)^{\kappa+1}
$$

where: $V_{s p 0}, p_{s p 0}$ - capacity and pressure in the initial state.

The basic non-linearity of the force characteristic $F(h)$ for the air spring results from the change of their effective surface $A_{e}(h)$. The principle of operation of 
bellows actuators for $p=$ const. it results that when the height $h$ of the spring increases, its volume $\mathrm{V}$ also increases, and then the efficiency of its surface $\underline{A}_{e}$ varies depending on its structure (type). In the case of a pneumatic spring, after filling it up to the pressure $p=$ const, its height $h$ increases, and then the force $F$ generated by the actuator decreases due to the reduction of its effective surface.

The force acting on the seat suspension system determined as follows [3]:

$$
F(h)=c_{s p}(h)\left(h_{0}-h\right)=\kappa \cdot p_{s p_{0}} \cdot A_{e} \frac{h_{0}-h}{h_{0}}\left(\frac{h_{0}}{h_{0}-h}\right)^{\kappa+1}
$$

For non-linear dependence:

$$
A_{e}=A_{e 0}+k_{1} h+\ldots+k_{n} h^{n}
$$

Substituting into equation (10) for $n=3$ to obtain the formula spring force of air non-linear change in the effective area $A_{e}$

$$
F(h)=\kappa \cdot p_{s p 0}\left(\cdot A_{e 0}+k_{1} h+k_{2} h^{2}+k_{3} h^{3}\right) \frac{h_{0}-h}{h_{0}}\left(\frac{h_{0}}{h_{0}-h}\right)^{\kappa+1}
$$

Where: $k_{1}, k_{2}, k_{3}$ - constant equation factors. The natural frequency $f_{0}$ of the air spring is:

$$
f_{0}=\frac{1}{2 \pi} \sqrt{\frac{\kappa \cdot p_{0 s p} \cdot g}{h_{0} \cdot p_{s p}}}
$$

The ratio of vibration amplitude transmission by air spring is defined as:

$$
T_{a}(f)=\frac{1}{\left(\frac{f}{f_{0}}\right)^{2}-1}
$$

Simulation studies for the proposed air spring model were carried out. Figures 3 and 4 show the model response for sinusoidal inputs for selected frequencies.

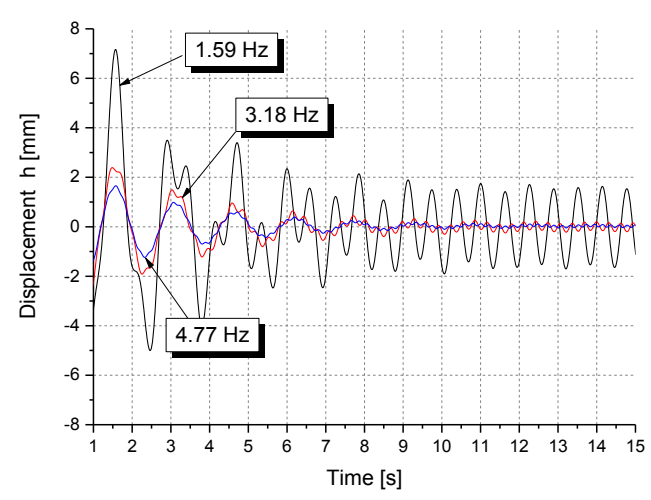

Fig. 3. Sinusoidal response of the air spring
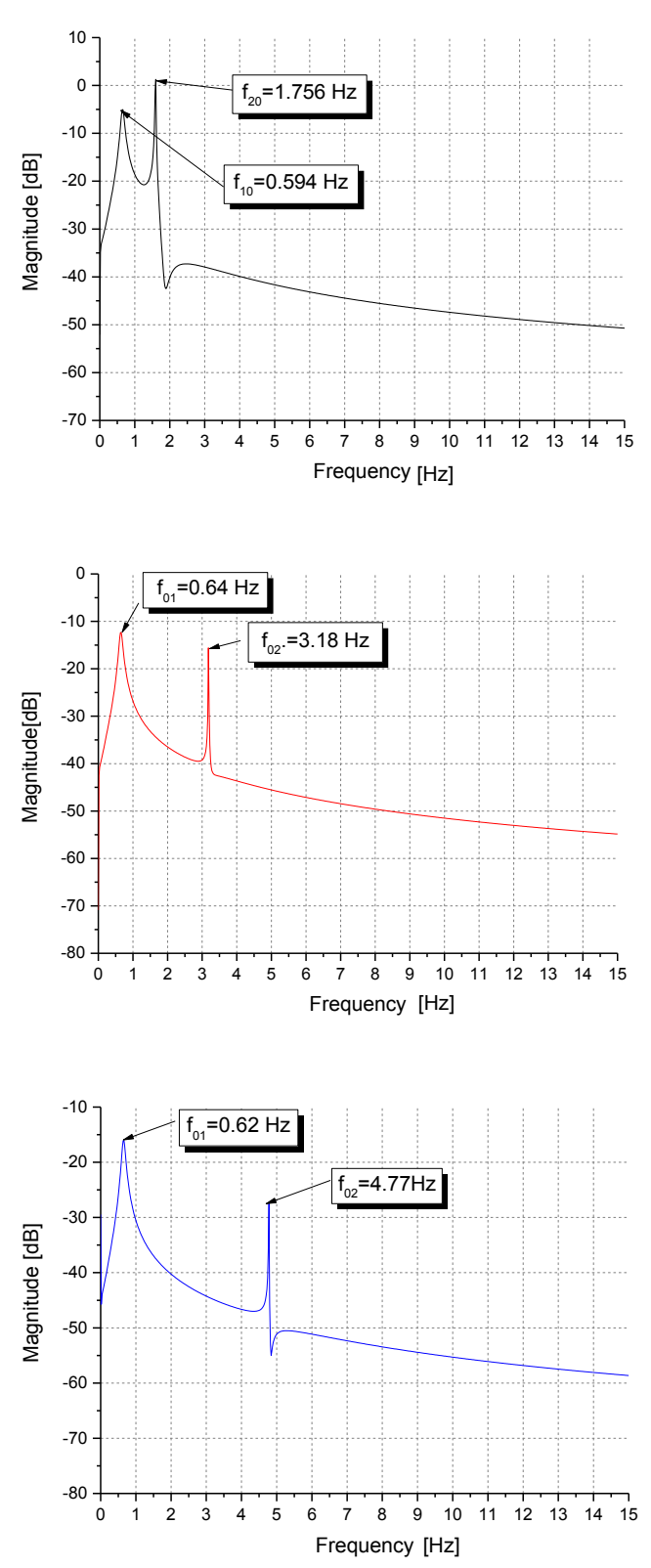

Fig. 4 Frequency response of the air -spring model: $f_{01}$ natural frequency, $f_{02}$-input frequency

In the simulation system of the model we observe a reduction of amplitude in the natural frequency range $f_{01}$ of air spring as well as the original input frequency range $f_{02}$.

\section{Experiments}

An experimental test stand for research on vibroinsulating properties of the working machine's seat built in the Department of Mechatronic Devices of the Kielce University of Technology is shown in figure 6. The basic element of the test stand is the vibration isolating seat base of the working machine (1). The lower part is attached to the vibrating table (4), and the upper one enables mounting the mass load (3). The vibratory table is driven by a pneumatic actuator controlled by a pneumatic proportional valve. This 
construction of the vibrator makes it possible to obtain vertical displacements in the range $(0-220 \mathrm{~mm})$ with a frequency of up to $5 \mathrm{~Hz}$. For non-contact measurement of relative seat displacement, the station was equipped with a laser sensor from the Baumer Electric OADM series (5). The control element of the air spring (2) is a pressure proportional valve from SMC type VEP3121-2 [10].

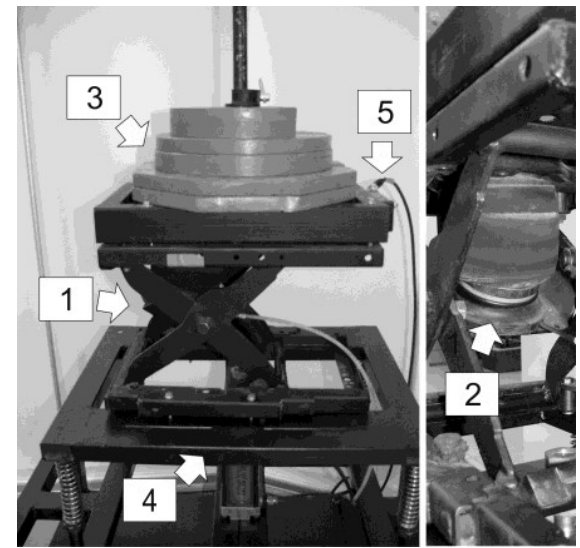

Fig. 6. Experimental test stand 1- suspension mechanism, 2 - air spring, 3 - load mass 4 - vibrating table, 5 - laser sensor

Figure 3 shows the timelines of the vibro-isolating mass $(65 \mathrm{~kg})$ for rectangular extortion and a pressure change in the air spring. The best vibro-isolating properties we obtained for the pressure ranging from 3.44 to 4.25 bar [10].

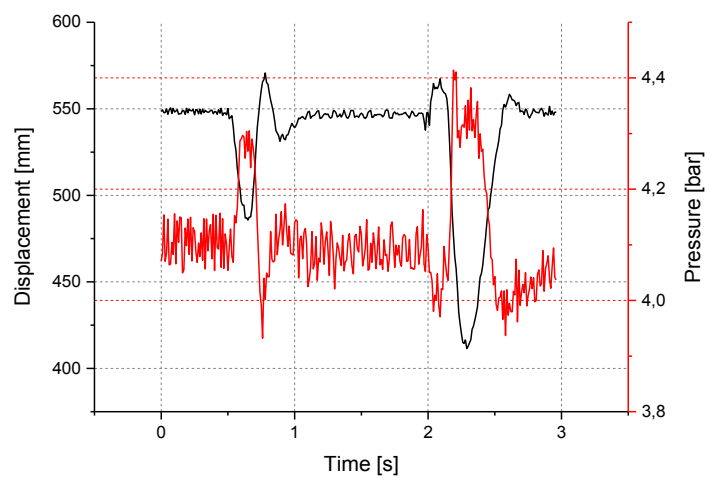

Fig.7. Vibro-isolating mass displacements with pressure change

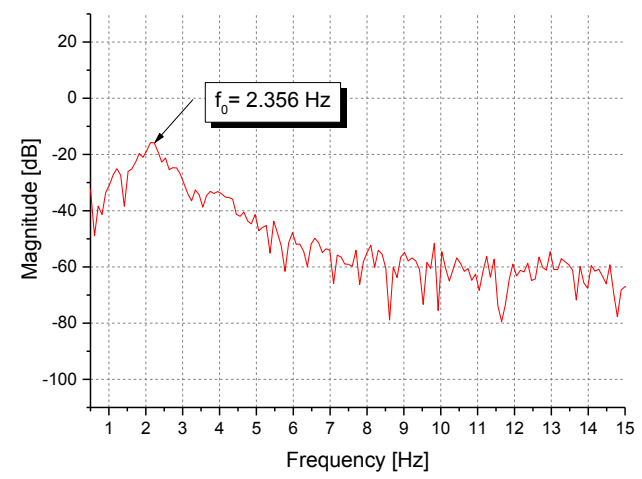

Fig. 8. Frequency response of the vibro-isolating system

\section{Conclusions}

An important element of pneumatic suspensions are air springs, in which during operation occur complicated physical phenomena that significantly affect their vibroinsulating properties. There are many dynamic factors that determine the effectiveness of vibroisolation in oscillating systems. One of them is the force generated by a bellows actuator. Proper estimation of the value of this force is the basic task of the good performance of semi-active and active suspension systems The simulation and experimental tests show that it is particularly important to change the effective surface of the applied pneumatic actuator. The air is a compressible medium, thanks to which the stiffness of the air spring is small, therefore the bellows actuators are used in systems with soft cushioning in the range to $3 \mathrm{~Hz}$.

\section{References}

1. W. Abbas, A. Emam, S. Badran, M. Shebl, and O. Abouelatta,. Control Autom., vol. 04, no. 02, pp. 199-205, (2013).

2. P. Beater, Pneumatic drives: system design, modelling and control. Springer, (2007).

3. R. Dindorf, Elastyczne aktuatory pneumatyczne. Wydawnictwo Politechniki Świętokrzyskiej, (2013).

4. E. Guglielmino, Semi-active suspension control: improved vehicle ride and road friendliness. Springer, (2008).

5. M. W. Holtz and J. L. van Niekerk, J. Sound Vib., vol. 329, no. 21, pp. 4354-4366, (2010).

6. N. Ketu, M. Demic, S. Muzdeka, and M. Krsmanovic, Vojnoteh. Glas., vol. 63, pp. 99-115, (2015).

7. I. Maciejewski, L. Meyer, and T. Krzyzynski, J. Sound Vib., vol. 329, no. 19, pp. 3898-3914, (2010).

8. J. E. Takosoglu, P. A. Laski, S. Blasiak, G. Bracha, and D. Pietrala, Int. Conf. Exp. F. Mech, pp. 814818, (2016).

9. G. Tora, Key Eng. Mater. vol. 542, pp. 219-231, Feb. (2013)

10. P. Wos, R. Dondorf, Int. Conf. Eng. Mech. pp. 1066-1069, (2017) 